\title{
Counterpropagating Matter Waves in Optical Lattices
}

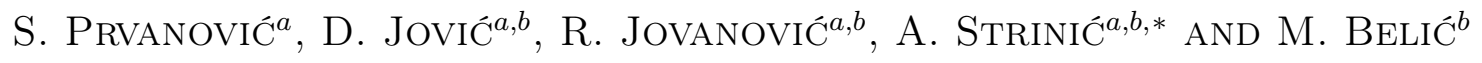 \\ ${ }^{a}$ Institute of Physics, P.O. Box 57, 11001 Belgrade, Serbia \\ ${ }^{b}$ Texas A\&M University at Qatar, P.O. Box 23874 Doha, Qatar
}

\begin{abstract}
An investigation of Bose-Einstein condensate in two-dimensional optical lattice potentials, formed by laser beams, is carried out. We are interested in the dynamics of Bose-Einstein condensate in a square optical lattice, where the periodic potential can lead to the stabilization of an otherwise unstable Bose-Einstein condensate. The behavior of Bose-Einstein condensate in optical lattices is described by the nonlinear Gross-Pitaevskii equation, which we treat numerically. By applying the Petviashvili iteration method, we demonstrate the existence of solitonic solutions in the case of counterpropagating matter waves, and analyze their stability.
\end{abstract}

PACS numbers: 03.75.Lm

\section{Introduction}

In a dilute boson gas at sufficiently low temperatures, particles can condense and, in this way, form a BoseEinstein condensate (BEC). Since 1995, when this phenomenon was first observed [1, 2], BECs have gained much attention, due to the fact that these condensates can be precisely manipulated in experiment $[3,4]$. On the other hand, properties of BECs show similarities with the physical systems studied in other branches of physics, such as nonlinear (NL) optics and NL wave theory. Similarities between these theories manifest themselves in dynamical equations used to describe the corresponding physical systems. More concretely, these theories share as their determining part the general NL Schrödinger equation (NLSE). In literature, there are several forms of NLSEs, and what they have in common is the term representing the nonlinearity. Within the mean-field model, the evolution of BECs is, in a pretty accurate way, described by the Gross-Pitaevskii equation (GPE). The NL term in this partial differential equation reflects the interatomic interaction in a diluted cold gas.

The investigation of BEC in two-dimensional (2D) potentials, formed by laser beams, has attracted much attention in the past few years [5-11]. Here, we are interested in the dynamics of BEC in an optical lattice, since the periodic potential can lead to the stabilization of an otherwise unstable BEC. We treat GPE numerically, by utilizing the Petviashvili iteration method (PIM) [13] to find localized solutions, and by propagating the solutions to check their stability.

\section{The model}

We study the interaction of two counterpropagating (CP) condensates that are initially confined to the so-

\footnotetext{
* corresponding author; e-mail: strinic@ipb.ac.rs
}

-called pancake shape $[8,12]$ and propagate head-on along the square optical lattice. In this manner we numerically consider the evolution of two BECs for which it is possible to take separated wave functions. The propagation of the forward $(F)$ and the backward $(B)$ BEC takes place in the longitudinal $z$ direction, while we study the intensity and the phase in the transverse $(x, y)$ plane. To this end, we employ the nonlinearly coupled 3D GPEs [12]. Finally, let us remark again that we seek the solutions of GPEs by utilizing PIM, and then we check the stability of such solitons by propagating them inside the lattice.

For the two $\mathrm{CP}$ components BEC, formed by the forward $(F)$ and the backward $(B)$ matter wave, we start from the dimensionless coupled GPEs

$$
\begin{aligned}
& -\mathrm{i} \partial_{z} F=\Delta F-V F-I F, \\
& \mathrm{i} \partial_{z} B=\Delta B-V B-I B,
\end{aligned}
$$

where

$$
I=|F|^{2}+|B|^{2}
$$

is the total intensity, and $F$ and $B$ are the forward and the backward wave functions. The external potential $V(x, y)$ is originating from the optical lattice and is given by the formula

$$
V=V_{0} \sin ^{2}[\pi(x+y) / d \sqrt{2}] \sin ^{2}[\pi(x-y) / d \sqrt{2}],
$$

where the potential depth $V_{0}$ is measured in units of the lattice recoil energy $E_{\mathrm{r}}=\hbar^{2} \pi^{2} /\left(2 m d^{2}\right), d$ is the lattice period and $m$ is the mass of the particles constituting BEC. In this treatment we neglect the external parabolic trapping potential, usually present in GPE, and concentrate on the influence of the optical lattice potential. We also assume an incoherent interaction between the two BEC clouds.

We are interested in the localized, solitonic solutions of Eqs. (1) and (2). Owing to their symmetry, the above equations suggest the existence of a fundamental vector soliton solution, of the form 


$$
\begin{aligned}
& F=u \cos \theta \mathrm{e}^{-\mathrm{i} \mu z}, \\
& B=u \sin \theta \mathrm{e}^{\mathrm{i} \mu z},
\end{aligned}
$$

where $\theta$ is an arbitrary projection angle (here $\theta=\pi / 4$ ), and $\mu$ is the chemical potential, or the propagation constant. Upon substituting these solutions into Eqs. (1) and (2), they transform into one, degenerate equation

$$
\Delta u+(\mu-V) u=u I \text {. }
$$

The solitonic solutions are found in this equation, using PIM [13]. The method itself just locates the solutions, with no regards to their stability. Of course, after finding the solitonic solutions, one should separately investigate their stability, e.g., by following their evolution according to the full system of Eqs. (1), (2). Here we will determine different classes of spatial vector solitons, and observe their stability.

\section{Numerical results}

For the chemical potential within the first gap, and for different topological charges (TCs) of the input waves, we found on-site and off-site solutions. An on-site vortex wave is positioned so that its center coincides with one of the lattice sites. An off-site vortex is positioned between the lattice sites. In Fig. 1 we show the intensity and phase distributions for the off-site (the first row) and the on-site (the second row) solitonic solutions, for fixed chemical potential $\mu=5$ and for different topological charges. It is seen that both types of solitons are fragmented and strongly pinned to the lattice sites. As one moves around the vortex, its phase jumps for $2 \pi$ times TC, as it should, but in a number of discrete steps.
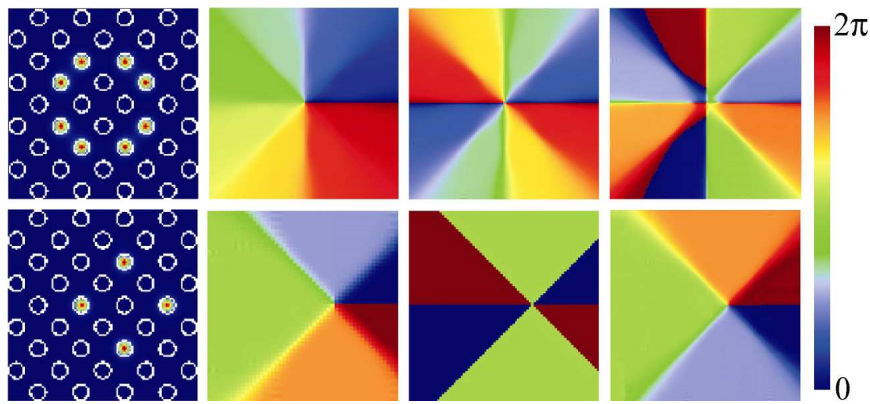

Fig. 1. Intensity and phase distributions of the off-site (the first row) and the on-site (the second row) solitonic solutions. The first column presents the intensity distributions, the remaining three the phase distributions, for different TCs. Parameters: $\mu=5, \mathrm{~V}_{0}=2$ and the input topological charges $\mathrm{TC}=1,2,3$, respectively.

Mutual interaction of the two CP BECs as they propagate, represented by the numerical solution of the above coupled GPEs, is depicted in Fig. 2 (for the input off-site CP solitons) and in Fig. 3 (for the input on-site CP solitons).

Numerical studies of the evolution of two CP solitonic solutions show how the two condensates influence each others during propagation. It is evident that for

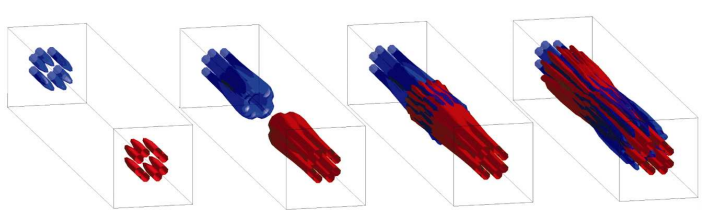

Fig. 2. Interaction of the off-site CP solitons (corresponding to Fig. 1, the first row) with $\mathrm{TC}=1$ during propagation

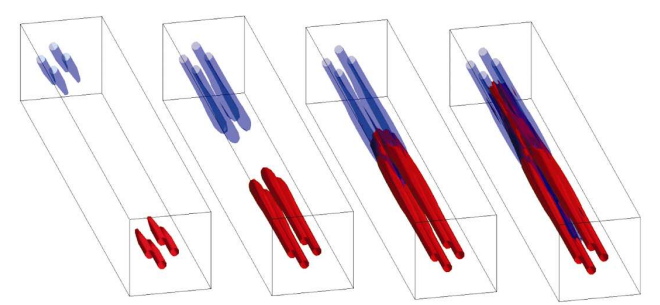

Fig. 3. Interaction of the on-site CP solitons (corresponding to Fig. 1 , the second row) with $\mathrm{TC}=1$ during propagation.

the chosen parameters they remain stable, but breathe slightly during propagation, forming the so-called soliton breathers.

\section{Conclusion}

We investigated the interactions of two-component BECs when illuminated by laser beams that form optical lattices, producing periodic external potentials. The behavior of BEC in an optical lattice is well described by the system of nonlinear Gross-Pitaevskii equations, which we solved numerically. By applying the Petviashvili iteration method, we investigated the existence of solitonic solutions in the case of counterpropagating matter waves, and analyzed their stability during propagation. We have displayed how the counterpropagating BECs evolve due to their mutual interaction and form stable breathing localized solutions, pinned by the lattice.

\section{Acknowledgments}

This study is supported by the Qatar National Research Foundation project NPRP 25-6-7-2 and by the Ministry of Science and Technological Development of the Republic of Serbia, under the project OI 141031.

\section{References}

[1] M.H. Anderson, J.R. Ensher, M.R. Matthews, C.E. Wieman, E.A. Cornell, Science 269, 198 (1995).

[2] K.B. Davis, M.-O. Mewes, M.R. Andrews, N.J. van Druten, D.S. Durfee, D.M. Kurn, W. Ketterle, Phys. Rev. Lett. 75, 3969 (1995).

[3] B. Eiermann, P. Treutlein, Th. Anker, M. Albiez, M. Taglieber, K.-P. Marzlin, M.K. Oberthaler, Phys. Rev. Lett. 91, 060402 (2003). 
[4] B. Eiermann, Th. Anker, M. Albiez, M. Taglieber, P. Treutlein, K.-P. Marzlin, M.K. Oberthaler, Phys. Rev. Lett. 92, 230401 (2004).

[5] H. Pu, L.O. Baksmaty, W. Zhang, N.P. Bigelow, P. Meystre, Phys. Rev. A 67, 043605 (2003).

[6] E.A. Ostrovskaya, Yu. S. Kivshar, Phys. Rev. Lett. 90, 160407 (2003).

[7] E.A. Ostrovskaya, Yu. S. Kivshar, Opt. Express 12, 19 (2004).

[8] E.A. Ostrovskaya, Yu. S. Kivshar, Phys. Rev. Lett. 93, 160405 (2004).
[9] T.J. Alexander, A.A. Sukhorukov, Yu.S. Kivshar, Phys. Rev. Lett. 93, 063901 (2004).

[10] T.J. Alexander, E.A. Ostrovskaya, Yu.S. Kivshar, Phys. Rev. Lett. 96, 040401 (2006).

[11] A.M. Dudarev, R.B. Diener, Q. Niu, J. Opt. B, Quantum Semiclassical Opt. 6, S231 (2004).

[12] B.J. Dabrowska, E.A. Ostrovskaya, Y.S. Kivshar, J. Opt. B, Quantum Semiclass. Opt. 6, 423 (2004).

[13] V.I. Petviashvili, Plasma Phys. 2, 469 (1976). 